\title{
Estratégias e resultados da prevenção do câncer bucal em idosos de São Paulo, Brasil, 2001 a 2009
}

\author{
Julie Silvia Martins, ${ }^{1}$ Sílvio Carlos Coelho de Abreu, ${ }^{1}$ Maria Ercília de Araújo, ${ }^{1}$ \\ Monique Marie M. Bourget, ${ }^{1}$ Fernanda Lúcia de Campos, ${ }^{1}$ \\ Marcus Vinícius Diniz Grigoletto ${ }^{1}$ e Fernanda Campos Sousa de Almeida ${ }^{2}$
}

Como citar Martins JS, Abreu SCC, Araújo ME, Bourget MMM, Campos FL, Grigoletto MVD, et al. Estratégias e resultados da prevenção do câncer bucal em idosos de São Paulo, Brasil, 2001 a 2009. Rev Panam Salud Publica. 2012;31(3):246-52.

RESUMO Objetivo. Descrever estratégias e resultados da campanha de diagnóstico precoce e prevenção do câncer bucal para a população com 60 anos ou mais desenvolvida desde 2001 no Estado de São Paulo.

Métodos. A partir de documentos publicados pelo Ministério da Saúde, Instituto Nacional de Câncer, Secretaria de Estado da Saúde de São Paulo, Fundação Oncocentro de São Paulo, Secretaria Municipal da Saúde de São Paulo, Faculdade de Saúde Pública da Universidade de São Paulo e Casa de Saúde Santa Marcelina, procurou-se evidenciar a problemática do câncer bucal e apresentar as principais estratégias utilizadas para desenvolver a campanha. Ainda, avaliou-se o impacto da campanha na incidência de casos novos de câncer bucal entre os examinados.

Resultados. Em 2001, foram examinados 90886 idosos, contra 629613 idosos em 2009. As estratégias identificadas foram: capacitação dos profissionais, desenvolvimento de material impresso para orientar os municípios sobre como conduzir a campanha, códigos e critérios a serem utilizados, orientações para a consolidação dos dados, estabelecimento de fluxos de referência, capacitação prática junto com o especialista na própria unidade de saúde a partir do reexame dos indivíduos que apresentavam alteração em tecidos moles e ampliação do número de serviços dirigidos ao diagnóstico bucal. Entre 2005 e 2009, houve redução significativa na taxa de casos confirmados de câncer bucal em cada 100000 examinados, de 20,89 para 11,12 ( $\mathrm{P}=0,00003)$.

Conclusões. A campanha trouxe benefícios à saúde bucal da população idosa. Pode ser ampliada para outros grupos etários e outras regiões do país e servir de subsídio para o desenvolvimento de ações de prevenção de câncer bucal em outros países, desde que respeitadas as características locais.

Palavras-chave Saúde bucal; neoplasias bucais; políticas públicas de saúde; programas de rastreamento; Brasil.

No Brasil, o Instituto Nacional de Câncer (INCA) estimou que, durante o ano de 2010, seriam registrados 14120 novos

\footnotetext{
Atenção Primária à Saúde Santa Marcelina, São Paulo (SP), Brasil. Correspondência: Julie Silvia Martins, julie@usp.br

2 Universidade de São Paulo (USP), Faculdade de Odontologia, São Paulo (SP), Brasil.
}

casos de câncer bucal. Para o Estado de São Paulo, no mesmo período, o INCA estimou 4120 novos casos de câncer bucal, com taxa bruta (para 100000 habitantes) de 15,19 para os homens e 4,02 para as mulheres (1). A figura 1 apresenta o coeficiente de incidência bruta padronizada por 100000 habitantes dos casos de câncer bucal no Município de São Paulo entre 1997 e 2003, conforme o Ministério da Saúde (2).

$\mathrm{O}$ grande problema que envolve o câncer bucal diz respeito à falta de diagnóstico precoce $(3,4)$, levando a sequelas ou mesmo à morte. Portanto, o rastreamento do câncer da cavidade bucal pode 
FIGURA 1. Coeficiente de incidência bruta e padronizada por 100000 habitantes dos casos de câncer bucal de acordo com grupo etário e sexo, Município de São Paulo, Brasil, 1997 a 2003

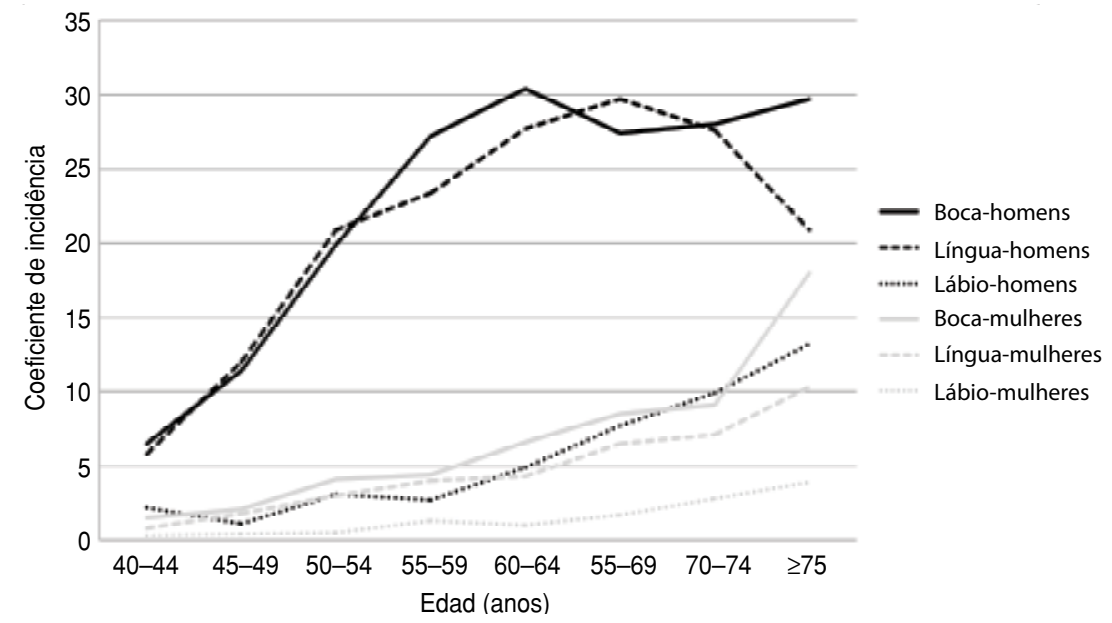

Fonte: Ministério da Saúde (2).

ser útil para detectar lesões pré-malignas e lesões de câncer, favorecendo o tratamento antes da instalação da doença e possibilitando uma melhor qualidade de vida a partir do diagnóstico precoce $(4,5)$.

Até o ano de 2001, embora algumas instituições realizassem, de forma isolada, ações para a prevenção do câncer bucal, não eram realizadas no Estado de São Paulo ações governamentais de grande amplitude com essa finalidade. A partir desse ano, a Secretaria de Estado da Saúde de São Paulo (SES-SP), em parceria com os municípios e organizações sociais ligadas à área da saúde, deu início à campanha de prevenção e diagnóstico precoce do câncer bucal (6), contando, a partir de 2005, com o apoio da Fundação Oncocentro de São Paulo (7). Tal campanha ocorre simultaneamente à campanha nacional de vacinação dos idosos contra a gripe, momento em que se aproveita a ida dos idosos à unidade de saúde para a imunização e realizam-se atividades educativas e preventivas, estimulando o autocuidado e o autoexame da cavidade bucal (6). A tecnologia de exame visual já demonstrou seu potencial de redução de mortalidade por câncer de boca em indivíduos de alto risco (5). A vinculação das duas campanhas é bem aceita e a inspeção da cavidade bucal é realizada com tranquilidade e sem constrangimentos para $69 \%$ dos examinados (8).

$\mathrm{O}$ objetivo do presente trabalho foi resgatar o percurso da campanha de prevenção do câncer bucal no Estado de São Paulo desde o seu início, em 2001, apontando as estratégias utilizadas e os principais resultados obtidos no período de 2001 a 2009.

\section{MÉTODOS}

As estratégias utilizadas em 9 anos de campanha de prevenção do câncer bucal em idosos no Estado de São Paulo foram levantadas a partir do exame de documentos publicados pela SES-SP e pela Casa de Saúde Santa Marcelina. Os dados numéricos foram obtidos do site oficial da Fundação Oncocentro de São Paulo (7). Como os dados referentes a 2010 ainda não foram finalizados, optou-se por não incluí-los.

Para verificar uma possível redução na incidência de casos novos de câncer bucal entre os examinados nos diferentes anos, utilizou-se o teste do quiquadrado $\left(\chi^{2}\right)$.

\section{RESULTADOS}

\section{Estratégias e implementação da campanha de prevenção do câncer bucal}

Foram identificadas as seguintes estratégias utilizadas pela campanha de prevenção do câncer bucal no Estado de São Paulo:

- aproveitamento de outra campanha consolidada (vacinação contra a gripe) para atingir o público-alvo;

- desenvolvimento de material impresso para guiar os municípios sobre a forma de conduzir a campanha, identificando códigos e critérios a serem utilizados e fornecendo orientações para a consolidação dos dados;

- capacitação dos profissionais;

- confecção de material educativo;

- realização de exames nos idosos;

- realização de grupos de orientação para os idosos sobre autocuidados e autoexames;

- estabelecimento de fluxos de referência;

- capacitação prática a partir do reexame dos indivíduos que apresentavam alguma alteração em tecidos moles junto com o especialista na própria unidade de saúde;

- ampliação do número de serviços dirigidos ao diagnóstico bucal (Centros de Especialidades Odontológicas, CEOs).

A campanha de prevenção do câncer bucal no Estado de São Paulo teve início em 2001, sendo oficialmente lançada durante o III Ciclo de Revisão em Saúde Bucal, evento promovido pela SES-SP em março de 2001 (9). Em 2002, a SES-SP passou a confeccionar uma apostila dirigida às Direções Regionais de Saúde (DRSs) e municípios, com orientações sobre os procedimentos a serem realizados e o preenchimento dos impressos para a totalização dos dados (10).

Um dos procedimentos preparatórios para a realização da campanha foi a capacitação dos profissionais envolvidos. Essa capacitação era realizada em nível local ou regional e podia envolver atividades práticas se houvesse recursos disponíveis. Nesse caso, era sugerido o envolvimento de faculdades de odontologia. Nos momentos de capacitação, discutiam-se os códigos e critérios a serem utilizados para o exame dos dentes e dos tecidos moles e para definir o uso e necessidade de prótese. Também eram discutidos o preenchimento dos impressos, a realização de atividades educativas com os idosos e os fluxos e encaminhamentos dos usuários, dando um enfoque especial ao diagnóstico bucal. Não foi prevista a realização de calibração dos profissionais durante a capacitação $(6,10,11)$. Com base em dados publicados por Morita et al. (12), estima-se que aproximadamente 10000 profissionais em todo o Estado estiveram envolvidos na campanha de 2009.

Desde o seu início, a campanha propunha o desenvolvimento de atividades educativas dirigidas ao autocuidado em saúde bucal, englobando higienização de dentes e próteses, orientações sobre 
os prejuízos provocados pelos hábitos nocivos, como tabagismo e alcoolismo, e estímulo ao autoexame da cavidade bucal, incluindo a distribuição de material educativo impresso $(6,10,11)$. Após a participação do idoso na atividade educativa, ele era convidado também a participar da inspeção da cavidade bucal, realizada pelo cirurgião-dentista, com o objetivo principal de verificar possíveis alterações em tecidos moles $(6,10,11)$. Os profissionais registravam dados como nome, endereço e telefone. Caso alguma alteração fosse constatada durante a inspeção visual, o participante era imediatamente agendado para reavaliação, diagnóstico e tratamento, na própria unidade de saúde ou em serviço de referência. Em relação ao registro da condição de tecidos moles, tem sido utilizada a classificação desenvolvida pelos técnicos da Casa de Saúde Santa Marcelina (13-15), que pode ser observada na tabela 1. Tal classificação foi recomendada pela SES-SP (16).

A proposta incluiu, desde o início, a utilização de planilhas para registro dos dados, permitindo a obtenção de informações sobre número de examinados, hábitos nocivos referidos, condição dentária, condição dos tecidos moles, uso e necessidade de prótese, condições das próteses utilizadas e necessidade de encaminhamento para serviços especializados. A planilha de registro incluía também espaço para a consolidação dos dados, permitindo que, ao final da campanha, cada unidade totalizasse os seus registros e os encaminhasse para a coordenação municipal. A coordenação municipal, por sua vez, encaminhava à DRS a consolidação dos dados do município. A DRS totalizava os dados de todos os municípios de sua região para a Coordenação Estadual da Campanha $(6,10,11)$.

TABELA 1. Códigos e critérios utilizados para avaliar os tecidos moles no exame bucal de idosos, São Paulo, Brasil, 2001 a 2009

\begin{tabular}{lcl}
\hline Classificação & Código & \multicolumn{1}{c}{ Critério } \\
\hline Baixo risco & 0 & $\begin{array}{l}\text { Indivíduo com tecidos moles sadios } \\
\text { Indivíduo com alterações em tecidos moles sem suspeita de malignidade } \\
\text { (alterações não listadas no código 2) }\end{array}$ \\
& 2 & $\begin{array}{l}\text { Indivíduo com alterações em tecidos moles com suspeita de malignidade: } \\
\text { úlceras indolores com mais de 14 dias de evolução, com bordas elevadas e } \\
\text { base ligeiramente endurecida ou não; lesões brancas ou enegrecidas com } \\
\text { áreas ulceradas; lesões avermelhadas com mais de 14 dias de evolução, com } \\
\text { contornos definidos e limites nítidos sugerindo eritroplasia; lesões vegetativas de } \\
\text { crescimento rápido (pápulas, nódulos), lisas, granuladas, verrucosas ou ulceradas }\end{array}$ \\
\hline
\end{tabular}

Fonte: Bourget (14).
Com a efetivação da campanha, surgiu a necessidade de um sistema de referência que possibilitasse o encaminhamento dos casos aos especialistas. As DRSs, bem como as coordenações municipais, empenharam-se nessa tarefa, buscando auxílio nas faculdades, não apenas como referência para atendimento especializado, mas também para a capacitação dos profissionais da rede básica $(6,10$, 11). A dificuldade de locomoção de muitos idosos para os serviços especializados e a falta de infraestrutura dos municípios para realizar de forma adequada esse deslocamento muitas vezes causava o abandono do tratamento (6). Como forma de contornar a situação, a Área Técnica de Saúde Bucal da SES-SP (ATSB-SES-SP) apresentou, no projeto referente à campanha de 2004, a experiência desenvolvida pelos técnicos da área de saúde bucal da Casa de Saúde Santa Marcelina (6), que consistia em agendar os usuários que apresentassem alguma alteração em tecidos moles em um dia específico, em que o especialista em diagnóstico bucal ia até a unidade básica de saúde e, juntamente com o cirurgião-dentista da atenção básica, reexaminava e discutia os casos, realizando os procedimentos necessários. Tal estratégia favoreceu a capacitação em serviço e permitiu reduzir o número de casos encaminhados (14). Em 2004, com o início da implantação dos CEOs em várias regiões do Estado, houve um incremento nos serviços de referência especializados, o que favoreceu o acesso dos usuários a esse nível de atenção.

Em decorrência da dissolução da ATSB-SES-SP, a partir de 2005, a campanha passou a ser desenvolvida pela Fundação Oncocentro, que, dispondo de mais recursos e de maior inserção nos serviços de atenção terciária, conseguiu

obter avanços no resgate de informações sobre a resolubilidade das ações desenvolvidas (7). Cabe salientar que a campanha, desde o seu início, não contou com recursos financeiros específicos, gerando como custo direto apenas a confecção de material impresso (apostilas descrevendo as formas de operacionalizar o projeto, planilhas para registro dos dados e folhetos educativos).

\section{Dados sobre câncer bucal}

A tabela 2 apresenta o número de municípios participantes da campanha de prevenção do câncer bucal, o número de idosos examinados e a cobertura de examinados entre os vacinados, de acordo com o ano $(7,17-19)$.

A tabela 3 apresenta os dados oficiais disponibilizados no site da SES-SP de 2001 a 2004 (17-19) e pela Fundação Oncocentro de São Paulo de 2005 a 2009 (7, 20), sendo possível verificar o número de idosos examinados nos diferentes anos, número e porcentagem de idosos que apresentaram alguma alteração em tecidos moles da cavidade bucal (indivíduos classificados com código 1 e 2 conforme tabela 1) nos diferentes anos, número de casos confirmados de câncer bucal através de biópsia e taxa de casos confirmados de câncer bucal a cada 100000 examinados a partir de 2005, pois tal dado só começou a ser coletado a partir desse ano.

Comparando-se os dados de 2005 e 2009, observa-se uma redução na taxa de casos confirmados em cada 100000 examinados, de 20,89 para 11,12. Aplicando-se o teste do $\chi^{2}$, observa-se uma diferença estatisticamente significativa na incidência de casos novos entre 2005 e $2009(P=0,00003)$.

\section{DISCUSSÃO}

Embora já existissem algumas iniciativas isoladas, a campanha de prevenção e diagnóstico precoce do câncer bucal promovida pela SES-SP em 2001 foi a primeira iniciativa que procurou agregar esforços em todo o Estado para o desenvolvimento de atividades educativas e preventivas relativas à prevenção do câncer bucal, envolvendo, desde o início, um grande número de municípios e ampliando a cobertura de indivíduos examinados ano a ano. A importância desse tipo de campanha tem sido amplamente reconhecida (21-25). 
TABELA 2. Participação na campanha de diagnóstico precoce e prevenção do câncer bucal em idosos, Estado de São Paulo, Brasil, 2001 a 2009

\begin{tabular}{lccc}
\hline Ano & $\begin{array}{c}\text { Municípios } \\
\text { participantes }\end{array}$ & $\begin{array}{c}\text { No. de idosos } \\
\text { examinados }^{\mathrm{a}}\end{array}$ & $\begin{array}{c}\text { \% de cobertura } \\
\text { sobre os vacinados }\end{array}$ \\
\hline $2001^{\mathrm{c}}$ & 334 & 90886 & 4,1 \\
$2002^{\mathrm{c}}$ & 434 & 142774 & 6,4 \\
$2003^{\mathrm{c}}$ & 512 & 226540 & 8,8 \\
$2004^{\mathrm{c}}$ & 490 & 238087 & 8,7 \\
$2005^{\mathrm{d}}$ & 317 & 253648 & 10,8 \\
$2006^{\mathrm{d}}$ & 509 & 360760 & 12,0 \\
$2007^{\mathrm{d}}$ & 503 & 435971 & 15,0 \\
$2008^{\mathrm{d}}$ & 539 & 480607 & 16,0 \\
$2009^{d}$ & 551 & 629613 & 18,5 \\
\hline
\end{tabular}

a Idosos = indivíduos com 60 anos ou mais.

b A campanha de saúde bucal é realizada concomitantemente com a campanha de vacinação contra a gripe.

c Dados da Secretaria de Estado da Saúde de São Paulo (17-19)

d Dados da Fundação Oncocentro de São Paulo (7).

TABELA 3. Alterações e casos confirmados de câncer bucal em cada 100000 idosos examinados, Estado de São Paulo, Brasil, 2001 a 2009

\begin{tabular}{|c|c|c|c|c|c|}
\hline \multirow[b]{2}{*}{ Ano } & \multirow{2}{*}{$\begin{array}{l}\text { No. de idosos } \\
\text { examinados }^{a}\end{array}$} & \multicolumn{2}{|c|}{$\begin{array}{l}\text { Idosos com alteração } \\
\text { em tecidos moles } \\
\text { (código } 1 \text { e } 2 \text { ) }\end{array}$} & \multirow{2}{*}{$\begin{array}{l}\text { No. de casos } \\
\text { confirmados de } \\
\text { câncer bucal }\end{array}$} & \multirow{2}{*}{$\begin{array}{l}\text { No. de casos confirmados } \\
\text { de câncer bucal/ } \\
100000 \text { examinados }\end{array}$} \\
\hline & & No. & $\%$ & & \\
\hline $2001^{b}$ & 90886 & 7028 & 7,8 & $\ldots$ & $\ldots$ \\
\hline $2002^{b}$ & 142774 & 13801 & 9,7 & $\ldots$ & $\ldots$ \\
\hline $2003^{c}$ & 226540 & 18059 & 8,0 & $\ldots$ & $\ldots$ \\
\hline $2004^{d}$ & 238087 & 20270 & 8,5 & $\ldots$ & $\ldots$ \\
\hline $2005^{e}$ & $253648^{f}$ & 22939 & 9,0 & $53^{f}$ & $20,89^{f}$ \\
\hline $2006^{e}$ & $360760^{f}$ & 30481 & 8,4 & $72^{f}$ & $19,95^{\dagger}$ \\
\hline $2007^{e}$ & $435971^{f}$ & 34346 & 7,9 & $54^{f}$ & $12,39^{f}$ \\
\hline $2008^{e}$ & $480607^{f}$ & 24280 & 5,0 & $52^{f}$ & $10,82^{f}$ \\
\hline $2009^{e}$ & 629613 & 28401 & 4,5 & 70 & 11,12 \\
\hline
\end{tabular}

a Idosos = indivíduos com 60 anos ou mais.

b Prevenção e diagnóstico precoce do câncer bucal - Relatório final (17).

c Prevenção e diagnóstico precoce do câncer bucal - Relatório final (18).

d Prevenção e diagnóstico precoce do câncer bucal - Relatório final (19).

e Relatórios das campanhas de prevenção e diagnóstico precoce do câncer bucal 2005/2009 (FOSP) (7).

${ }^{f}$ Dados reproduzidos de Bougert et al. (20).

A vinculação da campanha de prevenção e diagnóstico precoce do câncer bucal com a campanha nacional de vacinação do idoso contra a gripe foi uma importante estratégia para a captação dessa população, que se encontra na faixa etária com as maiores taxas de incidência de câncer bucal, assim como um fator facilitador para o contato da equipe de saúde bucal com a população idosa, já que muitos idosos consideram desnecessárias as visitas ao cirurgião-dentista, por serem totalmente desdentados (26). Os dados do Levantamento Epidemiológico em Saúde Bucal realizado em 2010 indicam que mais de 3 milhões de idosos encontram-se completamente edêntulos no Brasil (27).
Considera-se que a classificação desenvolvida pelos técnicos da área de saúde bucal da Casa de Saúde Santa Marcelina, testada no trabalho de Sartori (13) e posteriormente adotada para todo o Estado (16), favoreceu o desenvolvimento da campanha, pois facilita o rastreamento de alterações mesmo por cirurgiões-dentistas que não estejam acostumados a realizar diagnóstico bucal, possibilitando a seleção de casos que necessitem de uma atenção mais especializada.

O momento em que o cirurgião-dentista generalista e o especialista discutem e dão encaminhamento aos casos tem servido para capacitar os profissionais das unidades básicas de saúde (14). Essa estratégia, além de permitir que o usuário seja atendido mais perto de sua residência, favorece a capacitação dos profissionais da atenção básica em diagnóstico bucal e também facilita o contato entre os diferentes níveis de atenção. Os dados sugerem que os municípios que investirem na capacitação dos seus profissionais por meio dessa estratégia poderão reduzir o número de encaminhamentos desnecessários (14), oferecendo um atendimento de melhor qualidade a um menor custo. Os efeitos da utilização dessa estratégia de capacitação são bastante abrangentes, pois os cirurgiõesdentistas, uma vez capacitados e sensibilizados para a questão, incorporam esses conhecimentos na sua rotina de trabalho, favorecendo a prevenção e o diagnóstico precoce do câncer bucal em outros grupos etários. O contato entre os diferentes níveis de atenção parece estar permitindo que o cirurgião-dentista da atenção básica saiba para onde encaminhar cada um dos casos, quando necessário, evitando a perda de tempo, um fator decisivo no prognóstico.

Outro aspecto a ser ressaltado é a organização da campanha sem a disponibilização de recursos financeiros específicos. Isso só foi possível porque houve engajamento dos profissionais tanto da atenção básica quanto da especializada, assim como dos coordenadores municipais de saúde bucal e profissionais responsáveis pela área de saúde bucal nas DRSs. Esse esforço conjunto possibilitou a ampliação do número de examinados a cada ano, o estabelecimento de referências com serviços especializados, contato com universidades e serviços de atenção terciária, bem como a capacitação dos profissionais da atenção básica em diagnóstico bucal. Embora o número de indivíduos examinados seja bastante expressivo, o que chama atenção é a redução estatisticamente significativa de casos novos confirmados de câncer bucal, indicando uma possível efetividade da campanha.

Não se pode deixar de mencionar a importância dos CEOs, cuja implantação foi iniciada em 2004, contando com incentivos financeiros do governo federal. Os CEOs possibilitaram aumentar a disponibilidade de vagas para atendimento em serviços especializados em diagnóstico bucal justamente em um momento em que a campanha vinha ampliando o número de idosos examinados. O resultado foi uma resposta mais satisfatória às demandas identificadas. 
Os dados apresentados na tabela 3 mostram que, a partir de 2005, foi sendo reduzido o percentual de indivíduos que apresentavam alguma alteração em tecidos moles (código 1 e 2). Como a implantação dos CEOs começou em 2004, tal resultado pode estar refletindo a resolubilidade dos CEOs, que, através da remoção ou tratamento de lesões preexistentes, reduziu a incidência do câncer bucal na população-alvo da campanha. Tais resultados também podem estar refletindo um aumento da especificidade dos exames, uma vez que os profissionais, cada vez mais capacitados ao longo de 9 anos, diminuíram o número de exames falso-positivos, evitando que casos desnecessários fossem encaminhados ao especialista. Outro aspecto relevante a ser considerado é que esses usuários só tiveram acesso aos CEOs em decorrência da campanha. Portanto, o resultado provavelmente está atrelado a um efeito sinérgico entre a campanha e o CEO. Embora não existam dados referentes ao número de casos de câncer bucal confirmados antes de 2005, observou-se uma redução consistente no número de casos confirmados no período de 2005 a 2009 ( $P=0,00003)$. É preciso considerar que a participação dos profissionais em diversas capacitações e a experiência adquirida no decorrer dos anos tenderiam a aumentar a sensibilidade do exame e, consequentemente, favorecer a identificação de um maior número de lesões malignas; contudo, os dados indicam uma redução no número de lesões malignas, apontando, portanto, na direção da efetividade da campanha. Talvez uma possível explicação para essa redução possa estar relacionada à remoção de lesões pré-malignas em anos anteriores.

Um dos questionamentos referentes à campanha é o de que ela não atingiria todo o grupo etário de maior risco. Nesse sentido, é necessário considerar que o câncer bucal apresenta um aumento gradativo de incidência a partir dos 40 anos de idade, com os maiores coeficientes encontrados na população com 60 anos ou mais, como pode ser observado na figura 1 para o Município de São Paulo (2) - justamente a faixa etária envolvida na campanha. Portanto, apesar de não envolver todos os grupos etários que apresentam aumento na incidência da doença, a campanha certamente envolve os grupos etários que apresentam maior incidência.
É preciso envolver outros grupos etários nas ações de prevenção do câncer bucal; portanto, é necessário considerar as potencialidades da atenção básica para o desenvolvimento dessas ações, garantindo o acesso à informação relativa ao autocuidado e autoexame da cavidade bucal, esclarecendo os indivíduos quanto aos fatores de risco para o câncer bucal, como o uso de tabaco e/ou álcool e a exposição ao sol, entre outros, bem como garantindo o acesso universal à realização de exames periódicos, envolvendo dias e horários propícios aos trabalhadores.

Subramanian et al. (28), analisando os dados de um estudo longitudinal realizado na Índia, envolvendo quase 200000 pessoas, observaram que $95 \%$ dos casos de câncer bucal detectados ocorreram em indivíduos que faziam uso de tabaco ou álcool. Os autores concluíram que a melhor relação custo-benefício resultaria do rastreamento em indivíduos de alto risco (que fazem uso de tabaco ou álcool) a partir dos 35 anos. Losi-Guembarovski et al. (29), estudando pacientes com câncer bucal no Estado do Paraná, observaram que $93,4 \%$ eram fumantes e $76,9 \%$ consumiam álcool regularmente. McDowell (4) salientou a importância de realizar exames preventivos periódicos em todos os indivíduos, principalmente naqueles com 40 anos ou mais.

O fato de a Estratégia de Saúde da Família ter uma população cadastrada favorece a identificação e a abordagem dos indivíduos com maior risco (tabagistas, etilistas, pessoas que trabalham expostas ao sol, com doenças sistêmicas, entre outros), sendo propício o desenvolvimento de ações que procurem acompanhar mais de perto os indivíduos de alto risco, objetivando a prevenção do câncer bucal. Tendo em vista a grande ampliação que ocorreu no número de equipes de saúde bucal na Estratégia de Saúde da Família nesses últimos anos (30), e considerando o número de CEOs implantados (31), torna-se factível a adoção de tais medidas em boa parte do Brasil. A falta de acesso ao tratamento odontológico (26) não deve impedir o acesso ao exame de tecidos moles da cavidade bucal, que pode ser realizado em poucos minutos (4).

A luta contra o câncer bucal não deve envolver apenas os profissionais da área de saúde bucal (32). Todos os profissionais da saúde devem estar preparados para enfrentar a questão: os médicos, que frequentemente têm a oportunidade de examinar a cavidade bucal de seus pacientes, os enfermeiros, que se dedicam ao cuidado e à orientação de seus pacientes, e os agentes comunitários de saúde, que, durante as visitas domiciliares, têm uma boa oportunidade para abordar os malefícios causados pelo uso de tabaco e álcool e a importância do autoexame e de exames periódicos; mas, para isso, é necessário que estejam capacitados e sensibilizados para a questão.

Finalmente, é importante considerar que os dados aqui discutidos são oriundos dos serviços de saúde e, portanto, não mantêm todo o rigor metodológico de uma pesquisa científica. Outra limitação deste estudo é a falta de acompanhamento dos indivíduos examinados no decorrer dos anos. Os dados disponíveis são consolidados e não permitem acessar informações longitudinais dos indivíduos examinados. A propósito, o número de pessoas envolvidas e a importância da questão fazem desse tópico um campo bastante profícuo para o desenvolvimento de pesquisas, o que possibilitaria inclusive uma maior aproximação entre a academia e os serviços (33).

\section{CONCLUSÃO}

O presente artigo teve o objetivo de descrever as principais estratégias e resultados obtidos durante 9 anos de campanha de diagnóstico precoce e prevenção do câncer bucal no Estado de São Paulo, de forma a registrar essa experiência, que envolve milhares de idosos e um número expressivo de profissionais nos diferentes níveis, que, mostrando muito empenho, têm produzido uma mudança positiva na abordagem do câncer bucal no Estado de São Paulo. Os dados obtidos em 9 anos permitiram observar um aumento crescente de municípios envolvidos e de idosos examinados. Também mostram que os serviços vêm se organizando quanto à capacitação de recursos humanos e estabelecimento de referências, e que esses esforços parecem estar produzindo efeitos na redução da incidência de casos novos de câncer bucal na população-alvo. Conclui-se que a campanha tem trazido benefícios à saúde da população idosa, que tais estratégias poderiam ser ampliadas para outros grupos etários e outras regiões do país, e que poderiam inclusive servir de subsídio para o desenvolvimento de ações de prevenção de câncer bucal em 
outros países, considerando-se, evidentemente, as peculiaridades das diferentes realidades.
Agradecimentos. À Atenção Primária à Saúde Santa Marcelina, Faculdade de Odontologia da Universidade de São
Paulo (FOUSP) e Secretaria Municipal de Saúde de São Paulo pelo apoio ao desenvolvimento da pesquisa.

\section{REFERÊNCIAS}

1. Brasil, Ministério da Saúde, Secretaria de Atenção à Saúde, Instituto Nacional de Câncer, Coordenação de Prevenção e Vigilância de Câncer. Estimativas 2010: incidência de câncer no Brasil. Rio de Janeiro: INCA; 2010. Disponível em: http://www.inca.gov.br/es timativa/2010/index.asp?link=tabelaestados. asp\&UF=BR Acessado em 21 de dezembro de 2010.

2. Brasil, Ministério da Saúde, Instituto Nacional de Câncer; Secretaria de Estado da Saúde de São Paulo, Fundação Oncocentro de São Paulo; Secretaria Municipal de Saúde de São Paulo; Faculdade de Saúde Pública da Universidade de São Paulo, Departamento de Epidemiologia. Incidência de câncer no município de São Paulo, Brasil: 1997-2003; tendência no período 1969-2003; mortalidade por câncer no município de São Paulo, Brasil.

3. Epstein JB, Gorsky M, Cabay RJ, Day T, Gonsalves W. Screening for and diagnosis of oral premalignant lesions and oropharyngeal squamous cell carcinoma: role of primary care physicians. Can Fam Physician. 2008;54(6):870-5.

4. McDowell JD. An overview of epidemiology and common risk factors for oral squamous cell carcinoma. Otolaryngol Clin North Am. 2006;39(2):277-94.

5. Sankaranarayanan $R$, Ramadas $K$, Thomas G, Muwonge R, Thara S, Mathew B, et al. Effect of screening on oral cancer mortality in Kerala, India: a cluster-randomised controlled trial. Lancet. 2005;365(9475):1927-33.

6. Secretaria de Estado da Saúde de São Paulo, Coordenadoria de Planejamento em Saúde, Grupo de Avaliação Técnica de Saúde, Centro Técnico de Saúde Bucal. Prevenção e diagnóstico precoce do câncer bucal [apostila]. São Paulo: SES-SP; 2004.

7. Secretaria de Estado da Saúde de São Paulo, Fundação Oncocentro. Relatórios das campanhas de prevenção de câncer bucal 2005/2009. São Paulo: SES-SP; 2009. Disponível em: http://www.fosp.saude.sp.gov.br/ html/bucal_rel_2005_2010.html Acessado em 18 de dezembro de 2010.

8. Abreu AA, Fonseca JM, Teraoka MT, Pinheiro EPLR, Palma JC, Abreu SCC. Aceitação e satisfação do idoso em relação à campanha de prevenção do câncer bucal estar vinculada à vacinação contra a gripe, no Jd. Conquista em São Mateus, na zona leste do município de São Paulo, 2007 [resumo]. Saude Soc. 2008;17(Supl 1):53.

9. Secretaria de Estado da Saúde de São Paulo. Principais aspectos da atenção à saúde bucal do idoso - câncer bucal: fatores de risco, sinais e sintomas. III Ciclo de Revisão em Saúde Bucal; 2001 mar 22; São Paulo, SP, Brasil.

10. Secretaria de Estado da Saúde de São Paulo, Coordenadoria de Planejamento em Saúde, Grupo de Avaliação Técnica de Saúde, Centro Técnico de Saúde Bucal. Prevenção e diagnósdezembro de 2011. tico precoce do câncer bucal [apostila]. São Paulo: SES-SP; 2002.

11. Secretaria de Estado da Saúde de São Paulo, Coordenadoria de Planejamento em Saúde, Grupo de Avaliação Técnica de Saúde, Centro Técnico de Saúde Bucal. Prevenção e diagnóstico precoce do câncer bucal [apostila]. São Paulo: SES-SP; 2003.

12. Morita MC, Haddad AE, Araújo ME. Perfil atual e tendências do cirurgião-dentista brasileiro. Maringá: Dental Press; 2010.

13. Sartori LC. Rastreamento do câncer bucal: aplicações no Programa Saúde da Família [dissertação]. São Paulo: Universidade de São Paulo; 2004.

14. Bourget MMM, org. Programa Saúde da Família - saúde bucal. São Paulo: Martinari; 2006.

15. Sartori LC, Frazão P, Abreu SCC. Rastreamento do câncer bucal na Estratégia Saúde da Família. Rev Bras Saude Familia. 2006;7(12):32-7.

16. Secretaria de Estado da Saúde de São Paulo. Resolução 159/2007. Disponível em: http:// portal.saude.sp.gov.br/resources/ses/per fil/profissional-da-saude/grupo-tecnicode-acoes-estrategicas-gtae/saude-bucal/ documentos-tecnicos/resolucao_ss_159 de_24052007_e_anexos.pdf Acessado em 9 de

17. Secretaria de Estado de Saúde de São Paulo, Coordenadoria de Planejamento em Saúde, Grupo de Avaliação Técnica de Saúde, Centro Técnico de Saúde Bucal. Prevenção e diagnóstico precoce do câncer bucal: relatório final. São Paulo: SES-SP; 2002. Disponível em: http://portal.saude.sp.gov.br/resources / ses/perfil/profissional-da-saude/grupo-tec nico-de-acoes-estrategicas-gtae/saude-bucal/ artigos-e-teses / estudos-epidemiologicos / estudosepidemiologicos/prevencao_e_diag nostico_do_cancer.pdf Acessado em 9 de dezembro de 2011.

18. Secretaria de Estado da Saúde de São Paulo, Coordenadoria de Planejamento em Saúde, Grupo de Avaliação Técnica de Saúde, Centro Técnico de Saúde Bucal. Prevenção e diagnóstico precoce do câncer bucal: relatório final. São Paulo: SES-SP; 2003. Disponível em: http:/ / portal.saude.sp.gov.br/resources/ ses/perfil/profissional-da-saude/grupo-tec nico-de-acoes-estrategicas-gtae/saude-bucal/ artigos-e-teses / estudos-epidemiologicos / estudosepidemiologicos/prevencao_e_diag nostico_do_cancer_bucal_-_2003.pdf Acessado em 9 de dezembro de 2011.

19. Secretaria de Estado de Saúde de São Paulo, Coordenadoria de Planejamento em Saúde, Grupo de Avaliação Técnica de Saúde, Centro Técnico de Saúde Bucal. Prevenção e diagnóstico precoce do câncer bucal: relatório final. São Paulo: SES-SP; 2004. Disponível em http://portal.saude.sp.gov.br/resources/ ses/perfil/profissional-da-saude/grupo-tec nico-de-acoes-estrategicas-gtae/saude-bucal/ artigos-e-teses/estudos-epidemiologicos/ estudosepidemiologicos/prevencao_e_diag nostico_do_cancer_bucal_-_2004.pdf Acessado em 9 de dezembro de 2011.

20. Bougert MMB, Nogueira W, Abreu SCC, Martins JS. Epidemiologia do câncer: frequência no Brasil e na região leste do município de São Paulo e o trabalho da estratégia da saúde da família (ESF) em relação ao câncer. Em: Bifulco VA, Fernandes Júnior HJ, Barboza AB, coords. Câncer: uma visão multiprofissional. Barueri: Manole; 2010. Pp. 339-65.

21. Brasil, Ministério da Saúde, Secretaria de Atenção à Saúde, Departamento de Atenção Básica, Coordenação Nacional de Saúde Bucal. Diretrizes da política nacional de saúde bucal. Brasília: Ministério da Saúde; 2004.

22. Brasil, Ministério da Saúde. $3^{\text {a }}$ Conferência Nacional de Saúde Bucal: acesso e qualidade superando exclusão social; 29 jul-1 ${ }^{\circ}$ ago 2004; Brasília, DF, Brasil. Brasília: Ministério da Saúde; 2005.

23. Carta de Sorocaba. VI EPATESPO - Encontro Paulista de Administradores e Técnicos do Serviço Público Odontológico e V COPOSC - Congresso Paulista de Odontologia em Saúde Coletiva; 2002; Sorocaba, SP, Brasil. São Paulo: Secretaria de Estado da Saúde de São Paulo; 2002. Disponível em: http:/ / portal.saude.sp.gov.br/resources/ ses/perfil/profissional-da-saude/grupo-tec nico-de-acoes-estrategicas-gtae/saude-bucal/ artigos-e-teses/textos-do-epatespo/carta_de sorocaba_epatespo_-_2002.pdf Acessado em 9 de dezembro de 2011.

24. Carta de Marília. VII EPATESPO - Encontro Paulista de Administradores e Técnicos do Serviço Público Odontológico e VI COPOSC - Congresso Paulista de Odontologia em Saúde Coletiva; 2004; Marília, SP, Brasil. Marília: Secretaria de Estado da Saúde de São Paulo; 2004. Disponível em: http:// portal.saude.sp.gov.br/resources/ses / perfil/profissional-da-saude/grupo-tecnicode-acoes-estrategicas-gtae/saude-bucal/ artigos-e-teses/textos-do-epatespo/carta_de marilia_epatespo_-_2004.pdf Acessado em 9 de dezembro de 2011.

25. Carta de Peruíbe. VIII EPATESPO - Encontro Paulista de Administradores e Técnicos do Serviço Público Odontológico e VII COPOSC - Congresso Paulista de Odontologia em Saúde Coletiva; 2006; Peruíbe, SP, Brasil. São Paulo: Secretaria de Estado da Saúde de São Paulo; 2006. Disponível em: http://portal.saude.sp.gov.br/resources/ ses/perfil/profissional-da-saude/grupo-tecnico-de-acoes-estrategicas-gtae/saude-bucal/ artigos-e-teses/textos-do-epatespo/carta_de peruibe_epatespo_-_2006.pdf Acessado em 9 de dezembro de 2011.

26. Araujo CS, Lima RC, Peres MA, Barros AJD. Utilização de serviços odontológicos e fa- 
tores associados: um estudo de base populacional no Sul do Brasil. Cad Saude Publica. 2009;25(5):1063-72.

27. Brasil, Ministério da Saúde, Secretaria de Atenção à Saúde, Departamento de Atenção Básica, Coordenação Nacional de Saúde Bucal. SB Brasil 2010 - Pesquisa Nacional de Saúde Bucal. Brasília; Ministério da Saúde; 2010. Disponível em: http://189.28.128.100/ dab/docs/geral/apresentacao_SB2010.pdf Acessado em 20 de julho de 2011.

28. Subramanian $S$, Sankaranarayanan $R$, Bapat B, Somanathan T, Thomas G, Mathew B, et al. Cost-effectiveness of oral cancer screening: results from a cluster randomized controlled trial in India. Bull World Health Organ. 2009;87(3):200-6.
29. Losi-Guembarovski R, Menezes RP, Poliseli F, Chaves VN, Kuasne H, Leichsenring A, et al. Oral carcinoma epidemiology in Paraná State, southern Brazil. Cad Saude Publica. 2009;25(2):393-400.

30. Brasil, Ministério da Saúde, Secretaria de Atenção à Saúde, Departamento de Atenção Básica. Histórico de cobertura da Saúde Família [documento institucional "on-line"]. Brasília: Ministério da Saúde; 2010. Disponível em: http://dab.saude.gov.br/abnumeros. php\#historico Acessado em 21 de dezembro de 2010.

31. Costa JFR, Chagas LD, Silvestre RM, orgs. A política nacional de saúde bucal do Brasil: registro de uma conquista histórica. Brasília: Organização Pan-Americana da Saúde;
2006. (Série técnica Desenvolvimento de Sistemas e Serviços de Saúde; 11). Disponível em: http://189.28.128.100/dab/docs/pub licacoes/geral/serie_tecnica_11_port.pdf Acessado em 21 de dezembro de 2010 .

32. Almeida GCM, Ferreira MAF. Saúde bucal no contexto do Programa Saúde da Família: práticas de prevenção orientadas ao indivíduo e ao coletivo. Cad Saude Publica. 2008;24(9):2131-40.

33. Frazão P. Vinte anos de Sistema Único de Saúde: avanços e desafios para a saúde bucal. Cad Saude Publica. 2009;25(4):712-3.

Manuscrito recebido em 6 de abril de 2011. Aceito em versão revisada em 28 de setembro de 2011.

ABSTRACT Objective. To describe the strategies and results obtained by the early diagnosis and prevention of an oral cancer campaign targeting the population aged 60 years or older developed since 2001 in the state of São Paulo.

\section{Strategies and results of the oral cancer prevention campaign among the elderly in São Paulo, Brazil, 2001 to 2009}

\author{
Key words
}

Methods. The main strategies used to develop the campaign were described based on the review of documents issued by the Health Ministry, National Cancer Institute, São Paulo State Health Department, Oncocentro Foundation of São Paulo, São Paulo City Health Department, School of Public Health at the University of São Paulo (USP), and Santa Marcelina Health Care Center. The impact of the campaign on the incidence of new cases of oral cancer in the target population was evaluated.

Results. In 2001, 90886 elderly were examined vs. 629613 in 2009. The following strategies were identified: training of professionals, development of printed materials to guide municipal governments in developing the campaign and using standardized codes and criteria, guidelines for data consolidation, establishment of patient referral flows, practical training with a specialist at the basic health care unit after the followup examination of individuals presenting changes in soft tissues, and increase in the number of oral diagnosis services. Between 2005 and 2009, there was a significant reduction in the rate of confirmed cases of oral cancer per 100000 individuals examined, from 20.89 to $11.12(P=0.00003)$.

Conclusions. The campaign was beneficial to the oral health of the elderly and could be extended to include other age groups and regions of the country. It may also provide a basis for the development of oral cancer prevention actions in other countries, as long as local characteristics are taken into account.

Oral health; mouth neoplasms; health public policy; mass screening; Brazil. 